\title{
Electrochemical and Photographic Detection of Cavitation Phenomena within a Variable Frequency Acoustic Field
}

\author{
Peter R. Birkin, ${ }^{*} \dagger$ Christine L. Delaplace, ${ }^{\dagger}$ and Christopher R. Bowen \\ Department of Chemistry, University of Southampton, Southampton SO17 1BJ, U.K., and \\ SMC, DERA Farnborough, Farnborough, Hants GU14 OLX, U.K. \\ Received: July 10, 1998; In Final Form: September 28, 1998
}

\begin{abstract}
Electrochemical measurements of localized mass transfer effects within a cavitating liquid (sound field, 20$100 \mathrm{kHz}$ ) are reported. The contribution of acoustic streaming to mass transfer recorded at a microelectrode (Pt, $10 \mu \mathrm{m}$ diameter) is shown to be negligible in comparison to transient events. Active zones, within which high event density was observed, are shown to exist within the liquid. Transient events were recorded, within these active zones, at a frequency as high as ca. 4500/s, which was deemed the limit of resolution of the microelectrode and sort routine employed rather than a cavitation effect. These active zones are shown to be dynamic in nature. Average mass transfer coefficients within these active transient event zones of the order of $0.3 \mathrm{~cm} \mathrm{~s}^{-1}$ are reported as well as the influence of acoustic pressure on mass transfer within these active zones. Photographic evidence indicates the volume and dynamic nature of the active cavitation zones within the cell.
\end{abstract}

\section{Introduction}

The application of ultrasound to electrochemical systems has been studied in the literature for a variety of electrode geometrys and sound frequencies. ${ }^{1}$ The interest in this combination of sciences arises due to the unusual physical conditions and reactive intermediates which have been reported to exist within a high-intensity sound field. The application of such a sound field results in the generation of localized voids, or cavitation bubbles, which are thought to generate unusual conditions within the liquid. ${ }^{2,3}$ It has been reported that under such cavitating conditions extremely high transient temperatures and pressures may be generated. ${ }^{4-6}$ As well as these extreme conditions other high-energy physical effects have been observed. These include the acceleration of mass transfer of material throughout the liquid, the acceleration of mass transfer of material to a solid liquid interface, and the erosion of surfaces due to microjet and shock wave impact.

The investigation of electrochemical behavior within a cavitating liquid has understandably been associated with "dual activation" of chemical processes. ${ }^{7}$ Studies on the effects of ultrasound on many electrochemical processes can be found in the literature. Among these studies it has been shown that ultrasound can alter the product distribution of an electrosynthetic reaction, depassivate and erode an electrode surfaces, increase electrochemiluminescence, alter hydrogen loading in palladium, enhance stripping voltammetry, and alter gas evolution from electrode surfaces. ${ }^{8-18}$ Electrochemical investigations of mass transfer effects have been extensive and date back to as long ago as 1963 by Bard. ${ }^{19}$ Perusich and Alkire investigated the effect of a focused ultrasonic field on mass transport to an electrode. ${ }^{20,21}$ Dewald and Peterson employed a $20 \mathrm{kHz}$ ultrasonic source and constructed a hydrodynamically modulated

* To whom correspondence should be addressed. Fax: 01703676960. E-mail: prb2@soton.ac.uk.

University of Southampton.

$\div$ SMC, DERA Farnborough electrode by the use of a pulsed ultrasonic field. ${ }^{22}$ Zhang and Coury demonstrated that high pseudo-steady-state mass transfer with impressive mass transfer rates could be achieved by positioning an electrode within the plume of an operating ultrasonic horn. ${ }^{23}$ Reviews of the sonoelectrochemical research can be found in the literature. ${ }^{7,24-25}$

In much of the sonochemical literature large electrodes have been employed with low time resolution equipment to investigate the rate of mass transfer of the material to an electrode, alter chemistry, and investigate the effects on the solid surface. However, cavitation is known to be a highly localized and transient phenomenon ${ }^{2,3}$ and hence, to understand collective results, such as those obtained over a large electrode, investigation of individual effects produced by single cavitation bubbles within a sound field would seem complementary to existing investigations. It is with this ideology in mind that we have employed microelectrodes within a cavitating liquid to investigate individual current time transients arising from individual cavitation events. ${ }^{26,27}$ This methodology has several advantages when compared to the study of mass transfer with larger, more conventional electrodes. First, microelectrodes are small when compared to the cavitation events produced by a high-intensity sound field. As a consequence of the size of cavitation bubbles (a property dependent on the physical conditions within the liquid, particularly the acoustic pressure), compared to the size of the microelectrode and associated diffusion field, individual cavitation bubbles act as a convenient screen shielding the microelectrode from other cavitation events that will occur near the microelectrode at the same instant in time. As an example it is possible to calculate the maximum radius of an aqueous cavitation bubble within a $3 \mathrm{~atm}, 40 \mathrm{kHz}$ sound field as 127 $\mu \mathrm{m}$ from an initial $10 \mu \mathrm{m}$ bubble radius. This is larger than the diffusion field for a $10 \mu \mathrm{m}$ diameter microelectrode. Second, because of the diffusional characteristics of microelectrodes, relaxation of a compressed diffusion field back to a steadystate situation is relatively rapid, preparing the microelectrode for the next cavitation event. Third, because of the steady- 
state behavior of microelectrodes in static solution, analysis of the current time transients will be simplified in comparison to larger electrodes with non-steady-state mass transfer regimes.

Previously it was demonstrated how a microelectrode could be employed with a variety of redox probes in the presence of ultrasound to characterize mass transfer produced by ultrasound at $23 \mathrm{kHz} .{ }^{26,27}$ Cavitation phenomena at or near the microelectrode surface compressed or deformed the diffusion field set up by the microelectrode held at the mass transport limiting potential of the redox probe employed. These impacts resulted in high rates of transient mass transfer with mass transfer coefficients in the order of $1.5 \mathrm{~cm} \mathrm{~s}^{-1} .^{27}$ This study showed that reproducible results could be obtained, indicating that, within experimental error, the microelectrode was uniformly accessible at the current transient maximum. This enabled the study of fast electrode kinetics in the presence of ultrasound to be investigated. ${ }^{28}$

However, the majority of the investigations, including the microelectrode studies, into the effects of ultrasound on electrochemistry have been concentrated on single frequency ultrasound. This is partly a consequence of the ease of employment of ultrasonic horns or cell disrupters which produce prolific amounts of localized cavitation. Nevertheless some electrochemical studies have been performed with acoustic cavitation generated at higher acoustic frequencies $(>20 \mathrm{kHz})$. Trabelsi et al. investigated mass transfer data produced within a $561 \mathrm{kHz}$ acoustic sound field. ${ }^{29}$ They showed, with time averaged results recorded at a $1 \mathrm{~mm}$ diameter Pt electrode, that mass transfer coefficients of up to $0.0189 \mathrm{~cm} \mathrm{~s}^{-1}$ could be recorded within the $561 \mathrm{kHz}$ sound field employed. This study showed that mass transfer was heavily dependent on the position of the electrode within the cell. Again the study performed by Trabelsi et al. relied on an averaged measurement over a period of $5 \mathrm{~s}$ and employed an electrode which, due to its dimensions, could not attain true steady-state mass transfer behavior in the absence of forced convection.

The attraction to investigate the effects of increased frequency ultrasound on electrochemistry stems from studies which have shown that chemical effects may be enhanced at higher frequencies. ${ }^{30,31}$ As an example Petrier et al. showed that the rate of hydrogen peroxide production (associated with radical formation from within the collapsing cavitation bubbles) increased by a factor of ca. 6.5 when a $487 \mathrm{kHz}$ sound field was compared to a $20 \mathrm{kHz}$ source. ${ }^{30}$

In this paper an alternative method for the study of mass transfer to electrodes operating within an variable mid-acoustic frequency range $(20-100 \mathrm{kHz})$ is presented. Even though this is not as high as the ca. $500 \mathrm{kHz}$ work, it is aimed at investigating the comparitive effects of $20-100 \mathrm{kHz}$ ultrasound. This work answers for the first time the important questions of where are the high rates of mass transfer to be found within these types of acoustic fields, the magnitude of the mass transfer events involved, the frequency of particular events, and whether or not these regions are dynamic in nature.

This study shows that mass transfer coefficients within acoustic cells of the type used are severely localized with dynamic active regions within the sonoelectrochemical cell. Acoustic streaming is shown to have a negligible contribution when compared to transient events. Additional evidence for the inhomogeneity of the cavitation field is gained through photographic investigation of the cell in operation using luminol as a sonochemiluminescent probe.

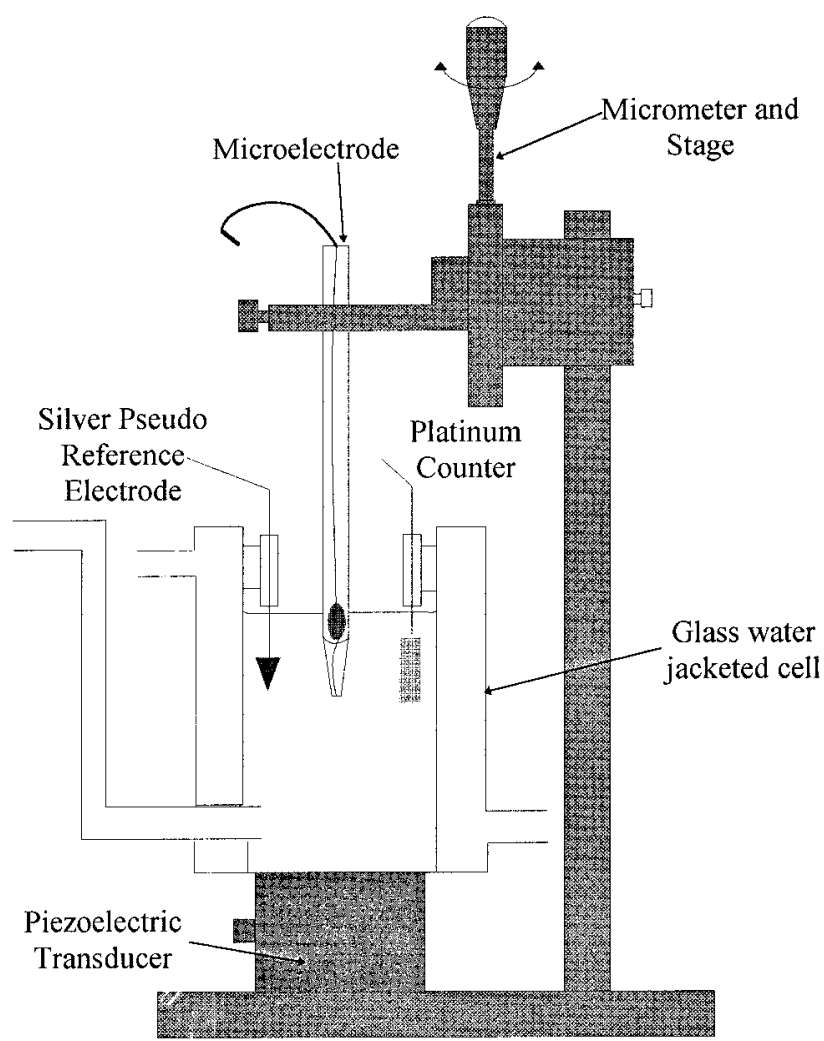

Figure 1. Schematic showing the experimental cell employed in the ultrasound/electrochemical experiments.

TABLE 1: Relationship between Power Input to the Transducer Operating at ca. $40 \mathrm{kHz}$ and the Acoustic Pressure Measured within the Cell ${ }^{a}$

\begin{tabular}{cccc}
\hline $\begin{array}{c}\text { voltage input to the } \\
\text { transducer } / V_{r m s}\end{array}$ & $\begin{array}{c}\text { pressure } \\
\text { amplitude/atm }\end{array}$ & $\begin{array}{c}\text { voltage input to the } \\
\text { transducer/ } V_{\text {rms }}\end{array}$ & $\begin{array}{c}\text { pressure } \\
\text { amplitude/atm }\end{array}$ \\
\hline $10-15$ & 0.8 & $35-45$ & 1.5 \\
$20-25$ & 0.9 & 50 & 1.7 \\
$25-30$ & 1.0 & &
\end{tabular}

${ }^{a}$ The cell contained $0.1 \mathrm{~mol} \mathrm{dm}^{-3} \mathrm{KCl}_{(\mathrm{aq})}$ at a height of $1.9 \mathrm{~cm}$ which corresponded to half a wavelength. The experiment was performed at $17^{\circ} \mathrm{C}$ under aerobic conditions.

\section{Experimental Section}

The experimental cell used to conduct cavitation experiments is shown in Figure 1. This cell was composed of a sandwich transducer (Morgan Matroc Ltd.) attached to an electrochemical glass cell with Quick set epoxy resin (RS). This cell was jacketed and had an internal diameter of $5 \mathrm{~cm}$. The experimental rig to produce cavitation consists of a programmable function generator (TG1010 from Thurlby Thandar instruments) used to generate a signal at the required frequency. The signal was passed into a Brüel \& Kjaer power amplifier 2713 before reaching the ultrasonic transducer. Acoustic pressure measurements were made with a Brüel \& Kjaer hydrophone 8103 and a Brüel \& Kjaer 2635 charge amplifier. The resultant AC signal was converted to DC, and the data were recorded on a PC. Typical acoustic pressures recorded in this manner are shown in Table 1. The electrochemical equipment consists of an inhouse-built potentiostat (eight channels), interfaced to a $486 \mathrm{PC}$ through a Computer Boards CIO-DAS08-AOL ADC/DAC interface card used as a virtual chart recorder for cyclic voltamograms or a National Instruments AT-MIO-16E-1 interface card (sampling rates up to $1 \mathrm{MHz}$ ) to record high time resolution sonoelectrochemical experiments. Electrochemical data were recorded with the aid of in-house-written software. 
A standard three-electrode electrochemical arrangement consisting of a Ag wire pseudo-reference electrode, a platinum gauze counter electrode, and a $10 \mu \mathrm{m}$ diameter Pt working electrode was employed. All measurements were recorded inside a Faraday cage in an effort to reduce electrical noise. The microelectrodes were made by sealing a $10 \mu \mathrm{m}$ diameter platinum wire into glass as described in the literature. ${ }^{32}$ They were initially exposed by being polished on emery paper, then polished further on $1 \mu \mathrm{m}$ and a $0.3 \mu \mathrm{m}$ alumina (Bueler) powder/ water slurry on velvet polishing pads (Bueler, Microcloth), and then finally rinsed with water. The microelectrode was positioned in the center of the cell, and the distance between the bottom of the cell and the tip of the microelectrode was adjusted using a micrometer and stage (Newport, BM 11.25 and UMR 5.25, respectively). This system had the ability to control the position of the microelectrode with a resolution of $10 \mu \mathrm{m}$ over a $2.5 \mathrm{~cm}$ travel. Experiments to determine the increase in mass transport due to ultrasound were performed under mass transport limited conditions at $-0.5 \mathrm{~V}$ vs Ag. The height of the solution within the cell was controlled and set to half a wavelength for a $40 \mathrm{kHz}$ sound field. This corresponded, under the conditions within our cell, to a depth of ca. $1.9 \mathrm{~cm}$. All solutions employed purified water (Whatman RO system). Hexaamine ruthenium(III) chloride (99\%) (STREM CHEMICAL) was used as a redox probe for cavitation experiments with $0.1 \mathrm{~mol} \mathrm{dm}^{-3} \mathrm{KCl}$ (HOGG Laboratory supplies) as supporting electrolyte. Solutions were sparged using argon for at least $15 \mathrm{~min}$ prior to electrochemical/cavitation experiments. The cell was connected to a water bath from Grant instruments Ltd. to control the temperature. The temperature was measured using a mercury thermometer and is reported in the appropriate figure legend.

Photographic data were recorded within a dark room. A Fuji Photo Optical Co. image intensifier CCD camera from Photonic Science and a Nicam video recorder were employed to record and capture the data, respectively. The cell contained $1.2 \mathrm{mmol}$ $\mathrm{dm}^{-3}$ luminol (3-aminophthalhydrazine, Aldrich) in a $0.05 \mathrm{~mol}$ $\mathrm{dm}^{-3}$ sodium carbonate (BDH, AnalR).

\section{Results and Discussion}

To investigate the effect of cavitation on mass transfer to an electrode, it is necessary to employ reversible electrode kinetics with a suitable redox system. In the course of this investigation several redox systems were investigated. It was found that ruthenium(III) hexaamine in $0.1 \mathrm{~mol} \mathrm{dm}^{-3} \mathrm{KCl}$ electrolyte was stable under the effects of cavitation and the variation of the steady-state current recorded at a microelectrode was negligible over the course of the experiments as long as the microelectrode remained intact within the cavitation field. This was tested frequently by recording cyclic voltammetry of the redox system at the microelectrode employed. If reversible voltammetry with a well-defined current plateau in the mass transfer region of the current potential plot (in this case between -0.3 to $-0.6 \mathrm{~V}$ vs Ag) was not observed, the microelectrode was repolished and the voltammetry repeated.

Characterization of the pressure field within the sonoelectrochemical cell employed in this investigation was achieved by pressure measurements employing a hydrophone. Figure 2 shows the pressure variation recorded at $0.5 \mathrm{~cm}$ from the base of the cell as a function of the applied frequency in the range $10-95 \mathrm{kHz}$. Clearly over this frequency range the sound field within the cell is complex in nature since, instead of a single frequency maxima at the transducer resonance (ca. $40 \mathrm{kHz}$ ), there are many pressure maxima observed within the entire 20-

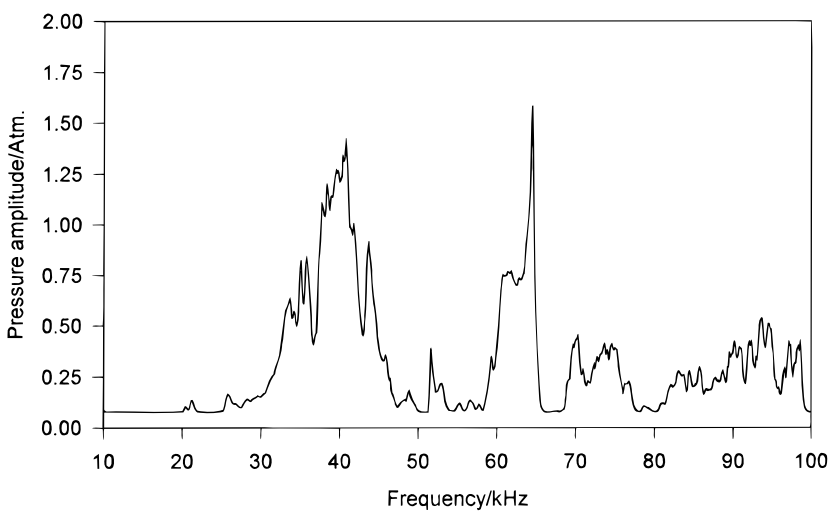

Figure 2. Plot showing the variation of pressure amplitude as a function of frequency. The experimental data were obtained using the hydrophone in a cell containing water at $25{ }^{\circ} \mathrm{C}$. The height of the solution corresponds to $3.9 \mathrm{~cm}$. The hydrophone was at $0.5 \mathrm{~cm}$ from the bottom of the cell.

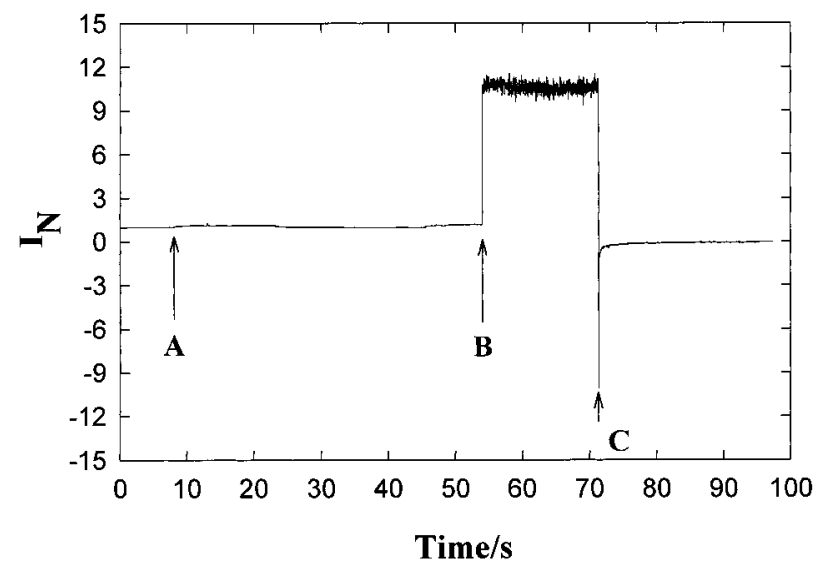

Figure 3. Plot showing the steady-state normalized current as a function of time recorded at a $10 \mu \mathrm{m}$ diameter Pt microelectrode. The cell contained $5 \mathrm{mmol} \mathrm{dm}^{-3}\left[\mathrm{Ru}\left(\mathrm{NH}_{3}\right)_{6}\right]^{3+}$ in a $0.1 \mathrm{~mol} \mathrm{dm}^{-3} \mathrm{KCl}$ aqueous solution at $25^{\circ} \mathrm{C}$. The solution height was adjusted to $2.0 \mathrm{~cm}$, and the electrode was $2.0 \mathrm{~mm}$ above the transducer. The markers are described in the text.

$100 \mathrm{kHz}$ range. A full description of the sound fields expected from transducers employed in electrochemical cells is beyond the scope of this paper and will be discussed elsewhere. ${ }^{33}$ However, it should be noted that the sound field generated within the cell depends on many factors. These include the temperature, the height of the liquid within the cell, the cell dimensions, the solvent viscosity, the transducer geometry, and the driving frequency. The dependence of the sound field on these physical parameters makes the investigation of the effect of one variable on mass transfer to a microelectrode sensitive to the exact physical conditions within the cell. This makes careful control of the physical conditions paramount to the achievement of reproducible results from experiments of this kind.

Low Time Resolution Results. Figure 3 shows the variation of the current recorded at a microelectrode within the sonoelectrochemical cell at low temporal resolution in the absence and presence of ultrasound (ca. 2 atm acoustic pressure at 38.75 $\mathrm{kHz}$ ). The microelectrode was held at the mass transfer limiting potential $(-0.5 \mathrm{~V}$ vs $\mathrm{Ag}$ ) between time $t=0$ and $C$. Initially between time $t=0$ and point $A$, the electrode was not exposed to ultrasound and hence a normalized current of 1 was observed. At point $A$ the transducer was turned on. However, the steadystate current remains approximately constant between point $A$ and point $B$ with slight "ripples" seen periodically. At point $B$ 
the current suddenly steps from a normalized current of 1 to a pseudo-steady-state current ca. 10 times larger (corresponding to a mass transfer coefficient, $k_{\mathrm{m}}$, of $0.12 \mathrm{~cm} \mathrm{~s}^{-1}$ ). During this period the microelectrode detected transient events, as indicated by the noise like characteristics of the current observed. Subsequent experiments employing higher time resolution equipment showed the transient nature of this current. It should be noted that, due to the time resolution of the data recording equipment employed in this experiment, an average signal was recorded. At point $C$, the potential of the microelectrode was changed from $-0.5 \mathrm{~V}$ to $+0.2 \mathrm{~V}$ vs $\mathrm{Ag}$ (a potential insufficient to reduce the redox probe) and no current was observed, clearly indicating that the signal detected prior to point $C$ was the result of increased mass transfer of the redox system to the microelectrode and not the result of an erroneous noise process. Figure 3 shows that when the microelectrode was initially irradiated with ultrasound, the effect of ultrasound on mass transfer to the microelectrode was negligible and only after ca. $40 \mathrm{~s}$ irradiation where transient events detected. These results were found to be typical, and hence two important conclusions can be drawn from the results shown in Figure 3. First, the absence of direct detection of transient cavitation events immediately after irradiation of the solution had commenced indicates that the volume detected by the microelectrode may not necessarily be active even though the entire solution was subject to ultrasonic irradiation. Hence the cavitation field within the cell can be described as inhomogeneous in nature. Second, in the absence of cavitation events (immediately after " $A$ ") the current recorded at the microelectrode was not increased significantly by acoustic streaming (slow movement of liquid as the result of sound adsorption within the sound field). Acoustic streaming can be related to the attenuation of sound through the media, and the attenuation coefficient in turn is related to the square of the frequency $(f)$; hence, an important factor in acoustic streaming is the frequency of the sound wave employed. To investigate the effect of acoustic streaming under optimized conditions, we measured the steady-state current at the highest possible frequency in our experimental setup (100 $\mathrm{kHz}$ ). In the absence of cavitation no significant enhancement in mass transfer to the microelectrode could be determined within the cell employed in our laboratory. It must be remembered that the mass transfer coefficient for the microelectrode and redox system employed is already $0.012 \mathrm{~cm} \mathrm{~s}^{-1}$, so the movement of liquid within the sound field as a result of acoustic streaming must be significant enough to be detected. Clearly under the conditions reported here this is not the case.

High Time Resolution Results. It has been shown in the previous section that transient events were not always detected at the microelectrode even though the solution was irradiated with ultrasound. However, by adjusting one of the three parameters such as frequency, power, or the position of the microelectrode in the cell, one can record current time transients detected by the microelectrode as soon as ultrasonic irradiation of the liquid is commenced. The data generated from such an experiment were recorded during a long period of time (ca. 5 s) with a high temporal resolution (sampling rate of 60-100 $\mathrm{kHz}$ ) in order to obtain meaningful statistical data. The mass transport coefficients were then calculated from the data recorded in this manner using eq 1 , where $n, F, A, c$, and $i_{\mathrm{AV}}$ represent the number of electrons transferred, Faraday's constant, the area of the electrode, the concentration of the redox species, and the average current recorded, respectively.

$$
k_{\mathrm{mt} A V}=\frac{i_{\mathrm{AV}}}{n F A c}
$$

To proceed further, it is clear that we must define enhanced mass transfer as the result of a cavitation event as a transient rise and fall in current recorded at the microelectrode. The position and magnitude of the cavitation event with respect to the microelectrode will affect the associated magnitude of the current time transient. Hence it is necessary to sort through the data, not only to determine large current time transients but also smaller current time transients.

To determine current time events, arising from cavitation enhanced mass transfer within the cell close to the microelectrode, software was developed to sort the data and calculate the maximum current, rise time, and frequency of each particular event. The procedure developed depended on the particular sampling rate employed in the experiment. As an example, when a sampling rate of $60 \mathrm{kHz}$ was employed with the ADC card, a moving gradient over six consecutive points was determined for the entire data set. This gradient was then averaged over three consecutive points to determine an average for the center point. The procedure was then moved along one point and repeated. This enabled the reduction of noise, always associated with differential signals, and enabled a subsequent sort routine to determine an event from analysis of the resultant differential. To determine whether a current time-transient as the result of a cavitation event had been detected by the microelectrode, the averaged differential signal was sorted to look for current maxima. This software procedure relied on a four consecutive rising averaged differential followed by four falling averaged differential to define an event. This procedure was necessary as simple analysis of rising and the subsequent falling current ${ }^{34}$ proved, in this case, to be inadequate. This was attributed to the high sample rate employed, which although enabling the resolution of closely spaced events, also picked up erroneous noise and subsequently made assignment of small events, in particular, difficult to achieve. Figure 4 shows a set of current-time transients recorded in the presence of ultrasound with the associated differential and peak assignment. The software developed was found to be the best compromise available at this time for the discrimination of both high- and low-current events with less than $10 \%$ error. This is typified in Figure 4 by the assignment of a missed current-time transient $(\square)$. This point, although missed by the routine, is rare in comparison with the correct assignment of the real events (-) with particular success with the small $\left(I_{\mathrm{N}}<5\right)$ current-time transients. The missed events are, in most cases, in a set of closely spaced current-time transients.

Figure 5 shows the average mass transfer coefficient recorded for a microelectrode held at a fixed position within the cell and irradiated with ultrasound of differing acoustic intensities. In this experiment, the microelectrode was positioned in the center of the cell and the frequency was chosen so that transient events were detected as soon as the irradiation of the liquid commenced at relatively low acoustic pressure (10 $V_{\mathrm{RMS}}$, ca. 0.8 atm). Current-time data were then recorded during $5 \mathrm{~s}$ for a variety of acoustic pressures with a sampling rate of $60 \mathrm{kHz}$. Below ca. $10 V_{\text {RMS }}$ no transient events could be observed, while above ca. $55 V_{\mathrm{RMS}}$ (ca. $2 \mathrm{~atm}$ ) it became necessary to alter the frequency of the sound field to record transient events. These results demonstrate that as the acoustic intensity was increased, the average mass transfer coefficient increased in an apparently linear fashion. Figure 6 shows that the increase in the average mass transfer coefficient appears to be related to the increasing density of cavitation events within the volume of liquid around 


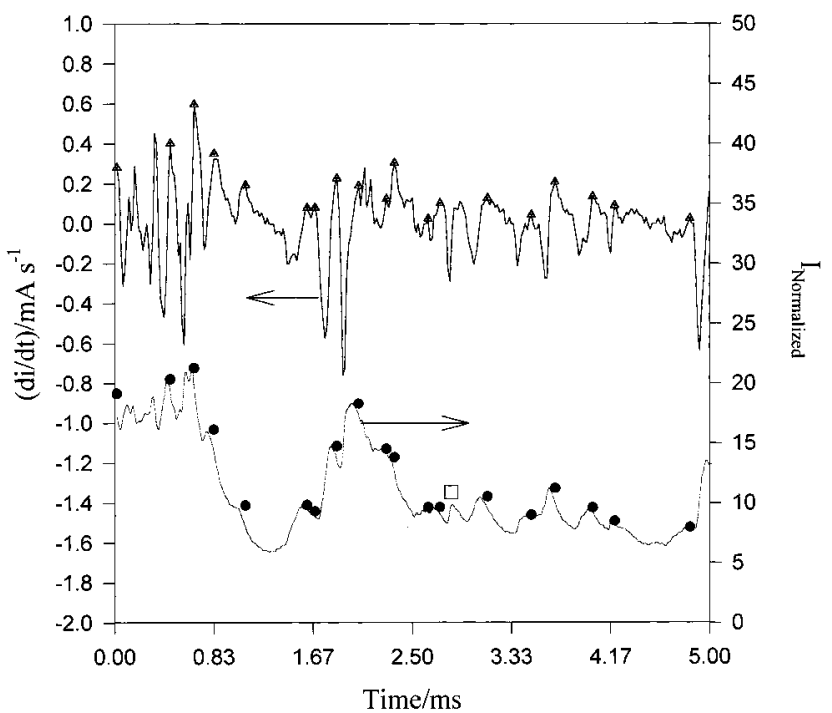

Figure 4. Plot showing a typical current-time trace ( $I_{\text {normalized }}$ representing the current normalized to the steady-state current recorded for the microelectrode in the absence of forced convection) and associated current differential signal $(\mathrm{d} i / \mathrm{d} t)$ recorded in the presence of ultrasound. $\Delta$ and - represent events determined using the software routine described in the text. ( $\square$ ) represents a missed event (see text).

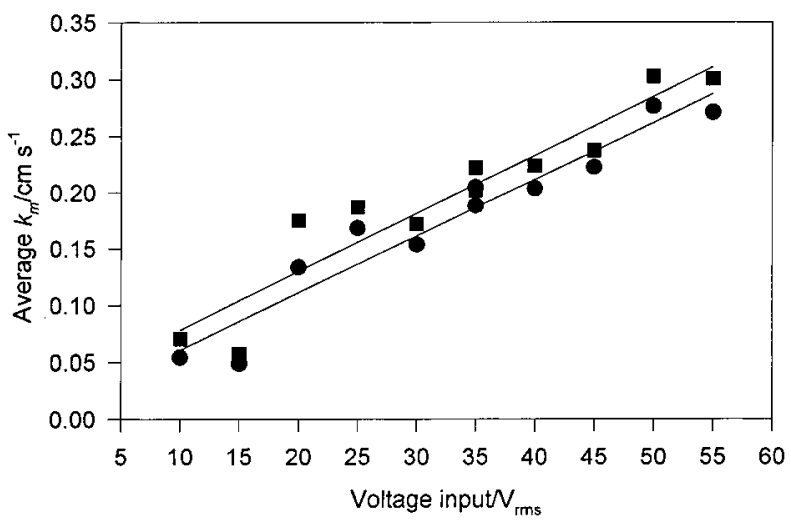

Figure 5. Plot showing the dependence of the average mass transfer coefficient as a function of the power applied to the transducer. represents the average current of all the data, while $\mathbf{r e p r e s e n t s ~ t h e ~}$ average current considering the transient events only. The data were recorded with a microelectrode to a sound source distance of $1.1 \mathrm{~cm}$. The solution contained $5 \mathrm{mmol} \mathrm{dm}{ }^{-3}\left[\mathrm{Ru}\left(\mathrm{NH}_{3}\right)_{6}\right]^{3+}$ in $0.1 \mathrm{~mol} \mathrm{dm}^{-3}$ $\mathrm{KCl}$ aqueous solution. The sound field was set at $39.6 \mathrm{kHz}$; the solution height was $1.93 \mathrm{~cm}$. The experiment was performed at $18{ }^{\circ} \mathrm{C}$ under anaerobic conditions. The electrode was held at $-0.5 \mathrm{~V}$ vs $\mathrm{Ag}$ throughout the experiment. The data were recorded at a sampling rate of $100 \mathrm{kHz}$.

the microelectrode. Initially as the acoustic pressure was increased, the number of events recorded per second also increased. However, as the power reaches ca. $35 V_{\mathrm{RMS}}$ the number of events reaches a maximum of ca. $4500-5000 / \mathrm{s}$. This limitation is related to the time resolution of the microelectrode employed and will be discussed later. The average mass transfer coefficient increases with increasing power beyond $35 V_{\mathrm{RMS}}$, indicating that even though the microelectrode and sort routine cannot distinguish more individual events, the overall current still increases. Figure 7 shows the distribution of the size of transient events recorded at 20,35, and $55 V_{\text {RMS. This shows }}$ that as the power increases, the number of events detected increases up until ca. $35 V_{\text {RMS }}$. However, the number of small events becomes negligible. The average event magnitude was found to increase, whereas the total number of events resolved remained fairly constant for powers greater than $35 V_{\mathrm{RMS}}$,

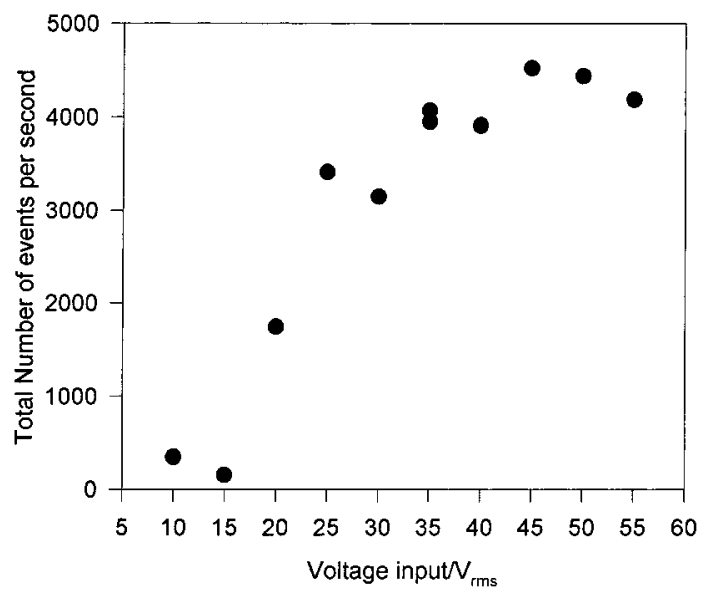

Figure 6. Plot showing the variation of the total number of resolved events as a function of the applied power. All other details are identical to those shown in Figure 5.

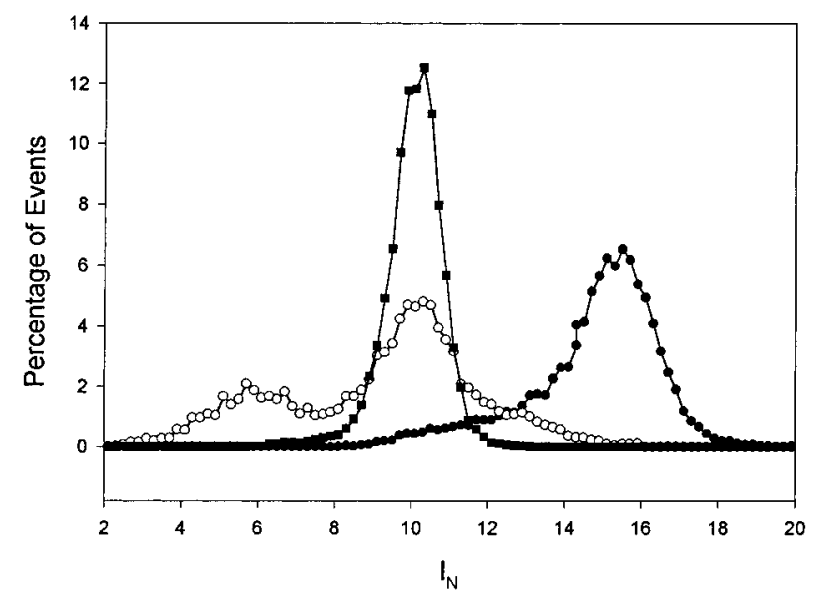

Figure 7. Plot showing the distribution of events at $20(\mathrm{O}), 35(\boldsymbol{\square})$, $50(-) V_{r m s}$. All other details are identical to those shown in Figure 5.

leading to the increase in mass transfer coefficient observed. The presence of two peaks on the $20 V_{\text {RMS }}$ data set can be attributed to the presence of clusters of closely spaced events, causing resolution of small events to become difficult (see later discussion). However, it should be noted that as the power increases, the magnitude of the highest current time-transient does not increase significantly. This is contrary to what would be expected if an increase in acoustic pressure produces an increase in bubble wall velocity on collapse during the process of cavitation.

During the course of these experiments it became clear that the frequency of events recorded could vary dramatically according to the exact physical conditions within the cell. Parts A and B of Figure 8 show two extremes of current-time traces recorded at a $10 \mu \mathrm{m}$ diameter microelectrode within the cell employed. Figure 8A shows a series of individual events resolved by the microelectrode, while Figure $8 \mathrm{~B}$ shows an almost pseudo-steady-state current recorded even at this relatively high temporal resolution. These two sets of results were obtained by collecting current-time data at a fixed position within the cell. Altering the driving frequency of the transducer by up to $100 \mathrm{~Hz}$ was sufficient to switch from one trace to the other, indicating the extreme sensitivity of the microelectrode to the local environment which itself is subject to the sound field employed. The microelectrode was then moved upward by $1 \mathrm{~mm}$ each time, and the same procedure applied. Figure 9 shows the variation in the average mass transfer coefficient, $k_{\mathrm{m} \mathrm{AV}}$, value recorded within the sonoelectrochemical cell as a 

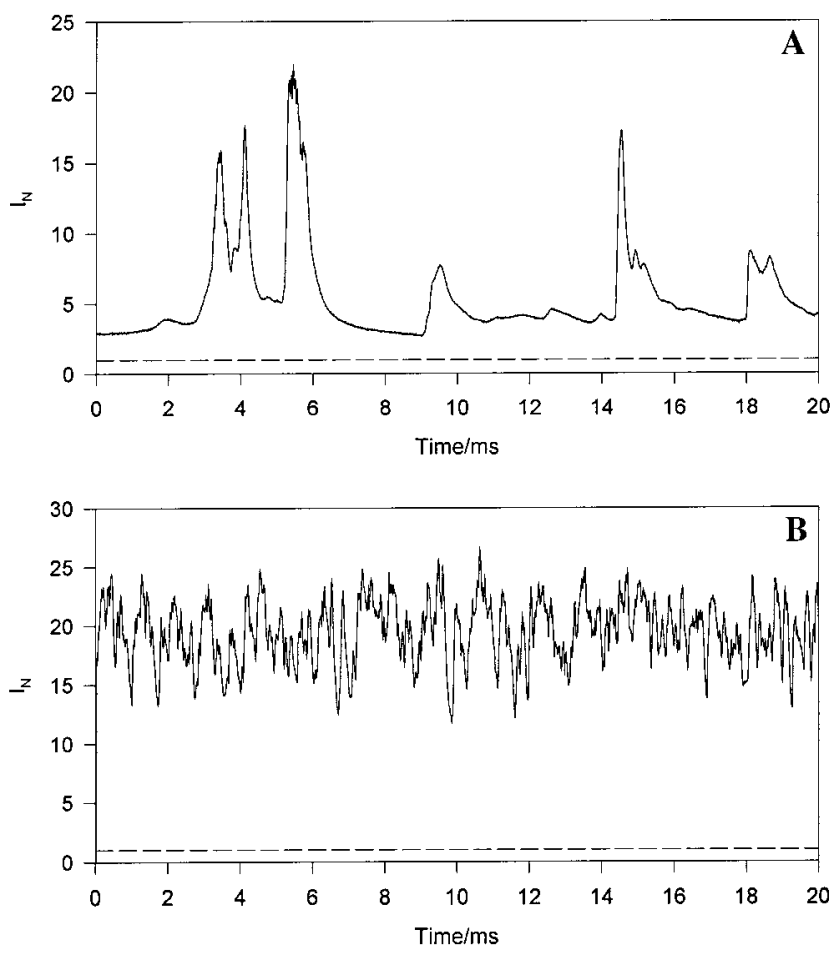

Figure 8. Plots showing the current response of a microelectrode taken from a single event data file (A) and a multiple event data file (B). The transducer frequency was ca. $39 \mathrm{kHz}$ but could be altered by up to $100 \mathrm{~Hz}$ between $\mathrm{A}$ and $\mathrm{B}$. The voltage input to the transducer was $40 V_{\text {ac }}(\mathrm{ca} .1 .5 \mathrm{~atm})$. The height of the solution in the cell was $1.9 \mathrm{~cm}$, and the temperature $16{ }^{\circ} \mathrm{C}$. The solution contain $5 \mathrm{mmol} \mathrm{dm}^{-3}$ ruthenium hexaamine(III) chloride and $0.1 \mathrm{~mol} \mathrm{dm}^{-3}$ potassium chloride. The electrode was placed at $1.1 \mathrm{~cm}$ from the base of the cell. The experiment was performed under anaerobic conditions.

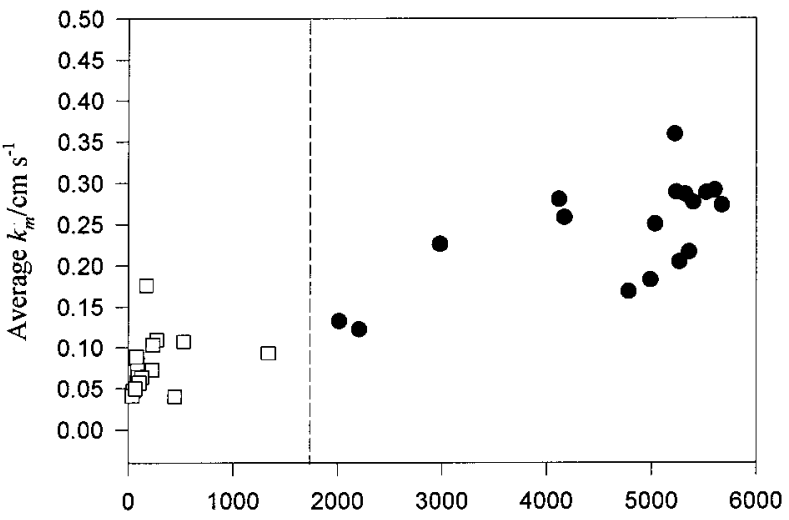

Number of events per second $/ \mathrm{s}^{-1}$

Figure 9. Plot showing the average mass transfer coefficient plotted as a function of the number of events recorded per second. All conditions are reported in Figure 8A,B except for a frequency shift of ca. $100 \mathrm{~Hz}$, and the position of the microelectrode was varied throughout the experiment. - represents the data files assigned as containing multiple events, while $\square$ represents the data files assigned as containing single events.

function of the number of events recorded per second. Two distinct groups of data can be seen: one at low event frequency and associated low average mass transfer coefficient, while the other is at high event frequency and high average mass transfer coefficient. These two groups can be associated with the two data sets where the microelectrode detects either sporadic individually temporally resolved cavitation events (Figure 8A) or almost continuous events (Figure $8 \mathrm{~B}$ ). These results indicate that there are areas within the cell which are highly cavitation

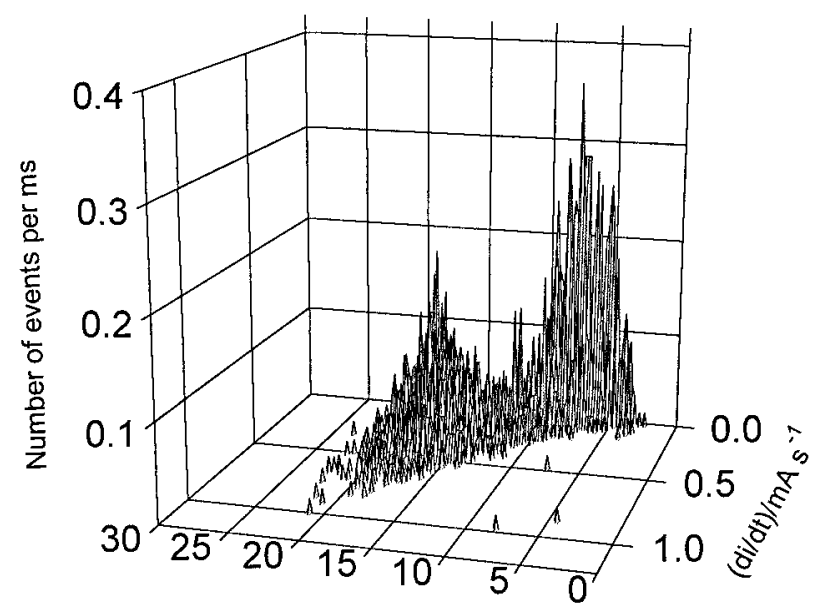

$I_{N}$

Figure 10. Plot obtained from a file assigned as containing single events. All conditions are reported in Figure 8A,B except for a frequency shift of ca. $100 \mathrm{~Hz}$.

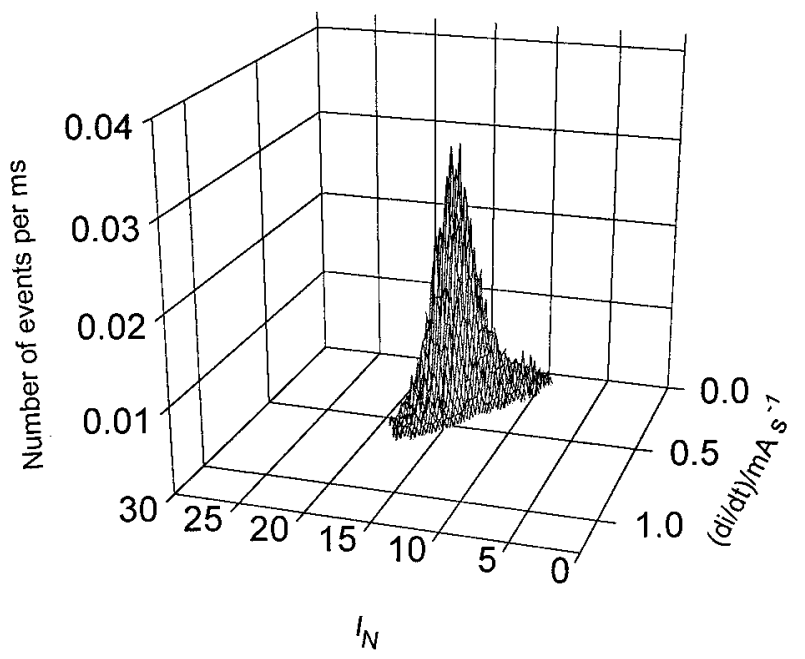

Figure 11. Plot obtained from a file assigned as containing multiple events. All conditions are reported in Figure $8 \mathrm{AB}$ except for a frequency shift of ca. $100 \mathrm{~Hz}$.

active, areas with no activity, and intermediate areas. These areas are mobile, and hence their position depends on the frequency and, thus, can be focused onto the microelectrode by fine adjustment of the sound field within the cell. The results shown in Figure 3 also indicate that at high acoustic powers these active cavitation zones are mobile and move through the liquid as indicated by the ripples seen between time $t=A$ and $t=B$. Only after point $B$ were transient events detected at the microelectrode. This indicates that the microelectrode was in an active cavitation zone only after some time from the start of irradiation of the liquid with ultrasound.

Distribution of Events. The data obtained from the microelectrode can be further analyzed to show the differences between the active zones within the cell. Figure 10 and Figure 11 show a plot of the normalized current as a function of the event frequency and the maximum rise time of each particular event for a data set with a majority of single events and multiple events, respectively. To explain the differences between the two sets of experiments, it is necessary to consider the resolution of the electrochemical sensor, in this case the microelectrode, placed within the ultrasonic field. The resolution of the microelectrode is related to its hemispherical diffusion field. Direct hits by cavitation events, whether transient collapse or 
some form of bubble motion, result in the highest expected current time event but with the lowest probability. Events at the extremity of the diffusion field, although the most probable, produce the smallest current enhancements. This physical situation would be expected to produce a distribution with the highest probability at low currents and low probability at high currents. The associated differential signal would be low at low currents but high at high currents. This physical situation should produce a distribution centered at low current running approximately diagonally across the differential scale. However, Figure 10 shows two clusters of events: one understandably having a high probability at low currents in agreement with the model and one at higher currents. Figure 11, which corresponds to multiple event data, shows only one distribution. This distribution is centered at higher currents than would be expected and occurs in the same region of the diagram as the second distribution in Figure 10. The explanation for these unusual distributions can be gained if one considers the resolution of the microelectrode in the context of the event density recorded when the microelectrode is within a cavitation active area of the cell. The resolution of the microelectrode will depend strongly on the type of event, the diffusion coefficient, and the size of the microelectrode. Considering the microelectrode and diffusion coefficient first, Bard et al. have shown by simulation that a microelectrode will reach a normalized current of 2.249 after a dimensionless time, $\tau$, of 0.1 following a potential step. ${ }^{35}$ The dimensionless time is related directly to the diffusion coefficient of the species employed and with an inverse square relationship to the radius of the microelectrode (e.g. $\tau=D t / a^{2}$, where $D, t$, and a represent the diffusion coefficient of the redox species employed, the real time, and the radius of the microelectrode, respectively). This implies that a smaller microelectrode detecting a species with a high diffusion coefficient will have a higher resolving power when compared to a large microelectrode detecting a species with a lower diffusion coefficient. Considering the dimensions of the microelectrode employed in this work ( $5 \mu \mathrm{m}$ radius) and the diffusion coefficient of the redox probe (ca. $5 \times 10^{-6} \mathrm{~cm}^{2} \mathrm{~s}^{-1}$ ), the time taken to reach 2.3 normalized current will be $5 \mathrm{~ms}$. This is clearly fast but does not approach the time scale of cavitation and the associated sound wave which is on the order of microseconds. If the volume of liquid where the microelectrode is situated is very cavitation active, then the response of the microelectrode cannot keep up with the cavitation events and essentially a pseudo-steady-state current will be recorded. As a consequence, small events cannot be recorded and a marked effect on the distribution of the events recorded in each data file can be observed. Figure 11 shows a distribution for a data file that can be assigned as corresponding to multiple events. This figure shows a single distribution centered at relatively high current but with a lower than expected differential signal. This is because the multiple event data consists of a relatively high current with few small events. Also since the events are so closely spaced, then the differential signal is also small. This is in contrast to the results shown in Figure 10, which demonstrate a data file containing a large proportion of single events. In this case the microelectrode is able to resolve single small events, and in agreement with the physical model the most frequent events occur at low current. However, the appearance of the second peak in the same region of the diagram as the peak shown in Figure 10 does indicate that even the data file defined as containing single events does contain some multiple or clustered events.

The results outlined above indicated that the volume of liquid within the cell is extremely inhomogeneous in terms of its cavitation activity. There are clearly zones within the liquid which may be mobile, as shown in Figure 3, that contain highly active cavitation. When the microelectrode encounters such areas, the cavitation density is such that the microelectrode may be saturated with events (greater than 4500/s) and record an almost steady-state current.

Photographic Data. To further confirm the results outlined above, photographic experiments were performed. Since it is difficult to record these environments by normal photographic means, it was necessary to image the bubble population by indirectly monitoring of radical production within the fluid. ${ }^{2}$ Radical production was monitored by employment of a chemiluminescent reaction. The chemiluminescent reaction of luminol (3-aminophthalhydrazine) was chosen as this has been previously employed within a cavitating fluid. ${ }^{3}$ This system should not be confused with light production from the cavitation process itself (a process known as sonoluminescence ${ }^{5}$ ).

To investigate the production of light within the cell, it was necessary to perform the experiments within a dark room. Figure 12A shows a top view of the sonoelectrochemical cell employed to measure mass transfer effects imaged using a luminol solution. In this experiment the frequency of the transducer was set at ca. $40 \mathrm{kHz}$ (a frequency close to the resonance frequency of the transducer). The equipment employed enabled semireal time capture of light production within the cell. These experiments showed that a column of light was produced by the cavitation process. It is possible from the photographic evidence using Figure $12 \mathrm{~A}$ as an example to measure the diameter of the light emitting area as $1.14 \mathrm{~cm}$. This in turn can be used, assuming a cylindrical volume within the cell, to calculate the active volume of the cell as ca. $4-6 \%$ of the entire volume of the cell at the acoustic intensity employed. This cavitation active volume remained fairly stable at low pressures $\left(<1.7 \mathrm{~atm}, 55 V_{\mathrm{RMS}}\right)$ but became increasingly more mobile at higher acoustic pressures or if the frequency of the transducer was altered slightly (see Figure 12B). These results are in strong agreement with the results obtained using a microelectrode. These types of bubble motion can be classed as streamers, which are well-known to exist in cavitating sound fields. ${ }^{6}$ It was also noted that nucleation of the streamer appeared to originate on the base of the cell. In the photographic and electrochemical experiments no special filtering processes where performed to remove particulate matter from the solution. However, the photographic evidence suggests that, in most cases, nucleation of the streamer occurs on the walls of the vessel.

Nevertheless the photographic data on its own only give on/ off (light or dark) information and are reliant on the efficiency of the radical production and coupled chemiluminescent reaction. Hence the combination of the photographic and electrochemical measurements can give valuable information on the position, dynamics, and density of cavitation within the liquid. It must also be remembered that the microelectrode itself may slightly perturb the sound field for direct result comparison at the same frequency to be made. However, photographic experiments showed that at least on the macroscopic scale the presence of the microelectrode did not appear to affect the position of light emission within the liquid.

The electrochemical and photographic data imply that within, for example, an ultrasonic bath or reactor, even if the acoustic field is well-defined, the population and streamer motion within the volume of liquid could have a dramatic effect on the results obtained.

It is probable that the mass transfer data shown in Figure 8B 

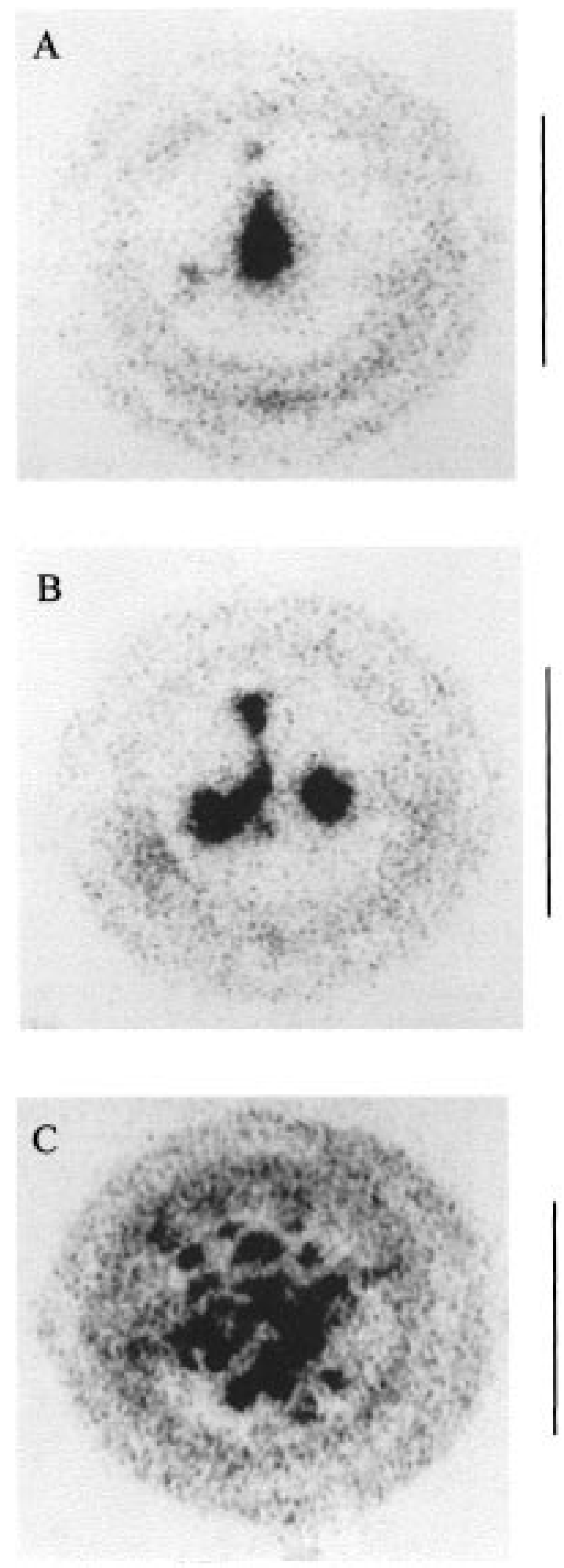

Figure 12. Pictures showing negative images of the top of an electrochemical cell, as described in the text, depicting bubble population as active light areas produced by sonochemiluminescence. The solution height in the cell corresponded to ca. $3.8 \mathrm{~cm}$. The solution consisted of $1.13 \times 10^{-3} \mathrm{~mol} \mathrm{dm}^{-3}$ luminol and $0.05 \mathrm{~mol} \mathrm{dm}^{-3}$ sodium carbonate. The transducer was operated at $40 \mathrm{kHz},(\mathrm{A})<1.7 \mathrm{~atm}$ and (B) $>1.7 \mathrm{~atm}$ and at $100 \mathrm{kHz}(\mathrm{C})$. The experiment was performed under aerobic conditions and at ca. $20^{\circ} \mathrm{C}$. The scale bar represents $5 \mathrm{~cm}$.

were the result of the microelectrode within a streamer. In such a position the microelectrode would be bombarded by bubble events. The high frequency of the events is clearly indicated by the pseudo-steady-state current recorded. Figure 8A was probably on the extremity of the streamer, and the microelectrode under these circumstances was able to record individual events.

Figure 12C shows the effect of increasing the sound frequency employed in the cell. In this case, at $100 \mathrm{kHz}$, the volume of cavitation active liquid appears to be increased when compared to $40 \mathrm{kHz}$. The light production indicates a more diffused sound field with radical production prevalent throughout the cell. However, the efficiency of the luminescent reaction as a function of sound frequency is not known. It has been suggested in the literature that at higher frequencies radical production, as reflected by hydrogen peroxide production and other sonochemical effects, may be increased. ${ }^{30}$ Alternatively another possible factor responsible for the increased rates of radical production at higher acoustic frequencies could be that the sound field at these frequencies is more diffuse and that the volume of active liquid within the cell is subsequently larger. Further investigation of this possibility is currently being investigated.

\section{Conclusions}

The results presented here demonstrate that electrochemical investigations of a cavitating media within a complex sound field are dependent upon the local environment and in particular the bubble population around the microelectrode at any instance in time.

The inherent complex and chaotic nature of cavitation within electrochemical cells result in the generation of active regions within the cell. In the absence of these active cavitation regions little or no enhancement to mass transfer to a microelectrode, which is already efficient, can be detected. This indicates that the contribution to mass transfer of acoustic streaming is negligible compared to cavitation within cells of this geometry.

Average mass transfer coefficients within the active cavitation regions appear to be related to the acoustic pressure, although it should be noted that this may be the result of increased event frequency. The data indicate that within the active cavitation zones of the cell the mass transfer coefficient can be as high as $0.3 \mathrm{~cm} \mathrm{~s}^{-1}$. A maximum cavitation density of 4500-5000 events/s has been recorded within the active zones within the liquid. This number was probably the limit of detection of the microelectrode/redox species and sort routine employed in this study.

Sonochemiluminescent experiments have shown that these active regions occupy ca. 4-6\% of the volume of the cell (in this case ca. $39 \mathrm{~cm}^{3}$ ) at $40 \mathrm{kHz}$ with much higher volumes active at higher frequencies.

These results imply that the bubble population as well as the acoustic field within the cell are important factors in determining the response of the electrode in the presence of ultrasound.

Acknowledgment. We would like to thank Dr. T. G. Leighton for the use of his light sensitive camera and the DERA for financial support for C.L.D.

\section{References and Notes}

(1) Marken, F.; Akkermans, R. P.; Compton, R. G. J. Electroanal. Chem. 1996, 415, 55-63.

(2) Leighton, T. G. The Acoustic Bubble; Academic Press: London, 1994, and references cited therein.

(3) Young, F. R. Cavitation; McGraw-Hill: London, 1989.

(4) Flint, B.; Suslick, K. S. Science 1991, 253, 1397-1399.

(5) Suslick, K. S.; Hammerton., D. A.; Cline, R. E. J. Am. Chem. Soc. 1986, $108,5641-5642$ 
(6) Flint, E. B.; Suslick, K. S. J. Am. Chem. Soc. 1989, 111, 69876992.

(7) Compton, R. G.; Eklund, J. C.; Marken, F.; Rebbitt, T. O.; Akkermans, R.; Waller, D. Electrochim. Acta 1997, 42, 2919-2927.

(8) Chyla, A.; Lorimer, J. P.; Mason, T. J.; Smith, G.; Walton, D. J. J. Chem. Soc., Chem. Commun. 1989, 9, 603-604.

(9) Compton, R. G.; Eklund, J. C.; Page, S. D.; Sanders, G. H. W.; Booth, J. J. Phys. Chem. 1994, 98, 12410-12414.

(10) Wark, A.; Crouch-Baker, S.; McKubre, M. C. H.; Tanzella, F. L. J. Electroanal. Chem. 1996, 418, 199-204.

(11) Walton, D. J.; Phull, S. S.; Bates, D. M.; Lorimer, J. P.; Mason, T. J. Electrochim. Acta. 1993, 38, 307-310.

(12) Compton, R. G.; Eklund, J. C.; Page, S. D.; Rebbitt, T. O. J. Chem. Soc., Dalton Trans. 1995, 389-393.

(13) Madigan, N. A.; Coury, L. A. Anal. Chem. 1997, 69, 5-15.

(14) Marken, F.; Eklund, J. C.; Compton, R. G. J. Electroanal. Chem. 1995, 395, 335-339.

(15) Whilcock, G. O. H.; Harvey, B. F. Ultrason. Sonochem. 1997, 4, $33-38$.

(16) Whilcock, G. O. H.; Harvey, B. F. Ultrason. Sonochem. 1997, 4, $23-31$.

(17) Marken, F.; Rebbitt, T. O.; Booth, J.; Compton, R. G. Electroanalysis 1997, 9, 19-22.

(18) Walton, D. J.; Burke, L. D.; Murphy, M. M. Electrochim. Acta 1997, 41, 2747-2751.

(19) Bard, A. J. Anal. Chem. 1963, 35, 1125-1128.

(20) Perusich, S. A.; Alkire, R. C. J. Electrochem. Soc. 1991, 138, 700707. 